\title{
The star formation history of the dwarf irregular galaxy SagDIG
}

\section{E. V. Held ${ }^{1}$, Y. Momany ${ }^{1}$, L. Rizzi ${ }^{2}$, I. Saviane ${ }^{3}$, L. R. Bedin ${ }^{4}$, M. Gullieuszik ${ }^{1,5}$, G. Bertelli ${ }^{1}$, E. Nasi ${ }^{1}$, M. Clemens ${ }^{1}$, M. R. Rich ${ }^{6}$ and K. Kuijken ${ }^{7}$}

\footnotetext{
${ }^{1}$ Osservatorio Astronomico di Padova, INAF, vicolo dell'Osservatorio 5, I-35122 Padova, Italy email: enrico.held,yazan.almomany,marco.gullieuszik, gianpaolo.bertelli,emma.nasi,marcel.clemens@oapd.inaf.it

${ }^{2}$ Institute for Astronomy, Univ. of Hawaii, 2680 Woodlawn Drive, Honolulu, HI 96822, USA email: rizzi@ifa.hawaii.edu

${ }^{3}$ European Southern Observatory, Casilla 19001, Santiago 19, Chile email: isaviane@eso.org

${ }^{4}$ European Southern Observatory, Karl-Schwarzschild-Str. 2, 85748 Garching, Germany email: lbedin@eso.org

${ }^{5}$ Dipartimento di Astronomia, Univ. di Padova, v.lo dell'Osservatorio 3, I-35122 Padova, Italy

${ }^{6}$ Department of Physics \& Astronomy, UCLA, Los Angeles, CA 90095, USA email: rmr@astro.ucla.edu

${ }^{7}$ Leiden Observatory, PO Box 9513, NL-2300 RA Leiden, The Netherlands email: kuijken@strw.leidenuniv.nl
}

\begin{abstract}
We present a preliminary reconstruction of the star formation history of the Sagittarius dwarf irregular galaxy (SagDIG), a dIrr galaxy at the border of the Local Group. SagDIG is a lively star-forming dwarf galaxy with very low metallicity and an abundant gas reservoir, for which deep HST ACS observations have been obtained (Momany et al. 2005). We have built synthetic color-magnitude diagrams in the intrinsic ACS bands F606W and F814W and compared them with our HST/ACS observations to derive the global star formation history of the galaxy. We find a broad episode of star formation between 3 and 8 Gyr ago, with a low-intensity tail of star formation at older ages, and a SF enhancement in the last Gyr. An upper limit to the old populations is set by modeling the old HB. Our modeling of the color-magnitude diagram also provides some constraints on the chemical enrichment history of SagDIG.
\end{abstract}

Keywords. galaxies: dwarf, Local Group, galaxies: stellar content, galaxies: evolution

The Sagittarius dwarf irregular galaxy (SagDIG) is a nearby dwarf galaxy characterized by on-going star formation in an extremely metal-poor environment, which makes it an ideal target to test locally the young galaxy hypothesis. Momany et al. (2005) have recently presented a study of the stellar populations in SagDIG based on deep colormagnitude diagrams (CMD) obtained from HST/ACS observations. Although the young stars are the most noticeable feature, Momany's et al. study showed that SagDIG began forming stars a long time ago, possibly more than 10 Gyr ago.

We present here a quantitative reconstruction of the star formation history $(\mathrm{SFH})$ of the galaxy based on the deep ACS data, using synthetic CMD techniques (see, e.g., Rizzi et al. 2003, and refs. therein). Figure 1 (left) presents the observed CMD with superposed a number of regions used to count stars in different evolutionary phases. In order to reconstruct the star-formation history of SagDIG, we generated a set of stellar populations in fixed age intervals, the duration of the episodes being a function of age. A fine grid with 200 Myr steps was used to map the SFH of the younger stars 

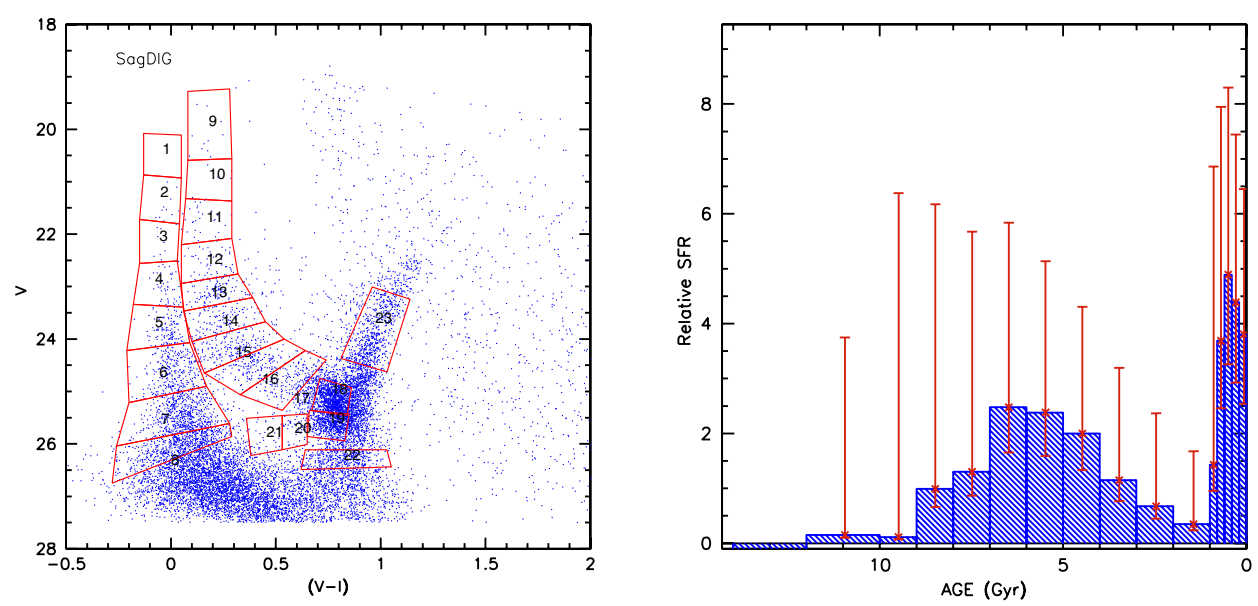

Figure 1. Left: The ACS color-magnitude diagram of SagDIG from Momany et al. (2005), with superimposed the CMD regions used to counts stars in differents evolutionary phases. Right: The star formation history of SagDIG from 14 Gyr ago to the present. Error bars represents $3 \sigma$ confidence intervals derived from a set of Monte Carlo realizations.

(in the last Gyr). Then we included stars in age bins of 1 Gyr at intermediate ages, between 1 and 10 Gyr ago. A coarser age resolution (2 Gyr) was used for older ages up to 14 Gyr ago. The age-metallicity relation was assumed as simple as allowed by the observational constraints. Momany et al. (2005) find a metallicity $[\mathrm{Fe} / \mathrm{H}] \sim-2.2$ for the red giant branch stars (uncorrected for age effects), while the appearance of the blue loops of helium-burning stars is consistent with $[\mathrm{Fe} / \mathrm{H}] \sim-1.7$. We therefore adopted a logarithmic increase in metallicity between $\mathrm{Z}=0.0001$ and 0.0004 , assuming that even the oldest stars had been promptly enriched to $Z=0.0001$. Finally, the observational errors and incompleteness in the different bands were included to obtain realistic simulations. Figure 1 (right) shows a plot of our preliminary best-fit SFH for SagDIG. Our results indicate a main star formation epoch between 2-9 Gyr ago, with a broad maximum about 6 Gyr, and a notable star formation enhancement in the last Gyr. Our best-fit synthetic CMD of SagDIG was compared with the observations. The overall agreement is good, even if not perfect. In particular, the distribution of young stars (main sequence and blue loops) is correctly reproduced with our choice of the metal enrichment history. Also, the number of old helium-burning stars is consistently reproduced apart from a slight disagreement in the red clump luminosity.

In summary, the dwarf irregular galaxy SagDIG shows a predominant star formation at intermediate ages, adding support to the evidence of suppression of star formation at early epochs in dIrr galaxies and a major star-formation episode about 5 Gyr ago. Thus, the SFH of SagDIG appears to be similar to that of the dwarf irregular IC 1613, discussed by Skillman et al. (2003). It is interesting to note that, in view of its extreme isolation and low metallicity, SagDIG can provide a paradigm for the evolution of dIrr galaxies in the absence of major interactions.

\section{References}

Momany, Y., Held, E. V., Saviane, I., et al. 2005, A\&A, 439, 111

Rizzi, L., Held, E. V., Bertelli, G., \& Saviane, I. 2003, ApJ, 589, L85

Skillman, E. D., Tolstoy, E., Cole, A. A., Dolphin, A. E., Saha, A., Gallagher, J. S., DohmPalmer, R. C., \& Mateo, M. 2003, ApJ, 596, 253 